\title{
Carnets
}

Revue électronique d'études françaises de l'APEF

Deuxième série - 2 | 2014

Frontières de la chronique

\section{Les Salons de Diderot : une chronique de la création artistique}

\section{Ana Fernandes}

\section{(2) OpenEdition}

Journals

\section{Édition électronique}

URL : http://journals.openedition.org/carnets/1295

DOI : 10.4000/carnets.1295

ISSN : 1646-7698

Éditeur

APEF

\section{Référence électronique}

Ana Fernandes, "Les Salons de Diderot : une chronique de la création artistique », Carnets [En ligne], Deuxième série - 2 | 2014, mis en ligne le 30 novembre 2014, consulté le 01 mai 2019. URL : http:// journals.openedition.org/carnets/1295; DOI : 10.4000/carnets.1295

Ce document a été généré automatiquement le 1 mai 2019.

\section{(c) (i) (8)}

Carnets est mis à disposition selon les termes de la licence Creative Commons - Atribution - Pas d'utilisation commerciale 4.0 International. 


\title{
Les Salons de Diderot : une chronique de la création artistique
}

\author{
Ana Fernandes
}

1 D'origine grecque (construit sur chronos, le temps) le terme évoque le souvenir d'un temps révolu. Mot à caractère savant, il renvoie à une construction culturelle élaborée où tenir chronique revient à inventer l'histoire, à prêter valeur à ce qui advient, surprend ou innove.

2 Admettant des formes diverses, les chroniques n'ont pas des préoccupations d'exactitude, cependant elles nous révèlent les époques et les gens, issues d'une production symbolique très élaborée tirée de l'identité de leurs auteurs.

3 Nous centrant sur une forme particulière de chronique au XVIII ${ }^{\mathrm{e}}$ siècle, celle des Salons de Diderot en tant que critique d'art, nous tenterons d'envisager comment la chronique est en même temps journal d'une vision picturale et jugement subjectif sur cette vision même.

4 Nous ne pouvons pas oublier que les Salons naissent d'une demande d'un des amis de Diderot qui dirigeait le journal La Correspondance littéraire : c'est le début des chroniques sur les expositions organisées tous les deux ans par l'Académie royale de peinture et de sculpture. Ces chroniques qui sont des commandes de Grimm prennent la forme de lettres. Sur le mode épistolaire, les Salons sont donc un genre hybride où les commentaires d'art sont insérés dans le tissu souple d'une lettre.

5 La Correspondance littéraire, en tant que journal manuscrit à quelques princes à travers l'Europe des Lumières pour leur rendre compte de l'actualité intellectuelle et littéraire de Paris, se présentait comme un vrai compte-rendu de la vie, de l'idéologie et de la société de l'époque. Diderot a eu une part importante dans la composition de ce journal.

6 Dans les Salons, Diderot dialogue à travers le tableau avec le peintre absent et devient reporter de ces séances culturelles pour un public qui ne les fréquentait pas.

7 La chronique porte un regard bien aigu sur la réalité et c'est précisément de ce point de vue que nous traiterons les Salons de Diderot: l'auteur voit l'Art comme un moyen 
d'interpréter le réel et le regard du critique devient ainsi une interprétation double sur le réel et la représentation de cette réalité-là.

Dans cet aperçu des Salons de Diderot, nous tenterons de passer en revue trois aspects de cette œuvre esthétique: l'esthétique de l'imitation, le spectateur dans l'œuvre et le langage pictural devenu langage littéraire car tous structurent le texte diderotien et rendent les Salons autant une critique d'art qu'une chronique.

\section{Une esthétique de l'imitation}

Dès ses premiers salons, Diderot remarque que les peintres « voient d'une façon et font d'une autre " (Diderot, 1876: X, 100) soulignant ainsi le peu de vraisemblance avec laquelle les peintres «imitent » la nature. On sait que dans le discours critique, même si on est attentif à la composition et à l'expression du tableau, il y a toujours l'explicitation d'une théorie de l'imitation en peinture, sur laquelle bien souvent Diderot s'appuie pour différencier les bons peintres des mauvais.

L'imitation se justifie par un certain approche du beau : la perception artiste de la nature ne peut prétendre saisir le beau idéal dans un objet, il faut l'esprit du peintre pour en différencier les parties belles et tenter de les imiter. C'est pour cela que tout tableau réussi est celui qui ne ressemble pas à un tableau, celui qui ne montre pas qu'il représente quelque chose. Ce qui est beau est avant tout ce qui « est bien fait », et cela ne s'apprend que par l'imitation de la nature. Cependant Diderot est conscient que tout dans la nature est soumis à la loi du changement et de la destruction. De ce fait, la « Beauté idéale » qu'il dénomme aussi « le modèle idéal », n'existe ni totalement ni partiellement dans la nature.

11 Comme le goût « exprime le choix » du peintre, alors tout tableau est subjectif, il est une prise de position personnelle s'opposant souvent à la position académique. Cette attitude s'explique par le fait que la plupart de ces artistes voient à travers le regard que l'Académie leur impose, c'est-à-dire qu'ils ne font que suivre les conventions, représentant la nature reformulée, embellie d'après le modèle antique que l'artiste doit connaître en profondeur. Diderot recherche toujours l'individualité sous-entendue par la création artistique dans les tableaux : ce n'est pas la manière d'imiter la nature qui fait la richesse d'un tableau, mais le mode individuel choisi pour l'exprimer. La théorie esthétique que Diderot expose dans le Salon de 1767 met effectivement la nécessité de l'imitation en avant, mais ce qui fait la beauté d'une œuvre, c'est dans l'individualité du peintre qu'on la trouve, non pas dans l'universalité de la nature, création de Dieu.

12 En ce qui concerne le modèle humain, l'Académie enseigne à corriger des formes imparfaites. L'élève doit conjuguer le modèle qu'il a sous les yeux avec les modèles qu'il a étudiés et le résultat ne sera qu'une contre-façon embellie de la nature et non la nature elle-même (Diderot, 1876 : XI, 153).

Diderot exige non seulement la vérité dans l'art de peindre un personnage mais encore dans la façon de rendre son attitude. Il se désole devant les pauvres produits de ces artisans tellement déformés par l'Académie qu'ils en ont perdu la faculté de voir la nature telle qu'elle est.

Le regard du philosophe est plus rigoureux que celui du peintre car il décèle l'attitude juste et le mouvement vrai qui dénoncent la fausseté d'une composition. À Vien il fait des suggestions pour rendre sa Psyché plus naturelle : 
Elle devrait avoir la bouche entr'ouverte et craindre de respirer. C'est son amant (... ) qu'elle voit pour la première fois, au hasard de le perdre. Quelle joie de le voir et de le voir si beau! Oh! que nos peintres ont peu d'esprit! qu'ils connaissent peu la nature ! La tête de Psyché devrait être penchée vers l'Amour ; le reste de son corps porté en arrière, comme il est lorsqu'on s'avance vers un lieu où l'on craint entrer et dont on est prêt à s'enfuir ; un pied posé et l'autre effleurant la terre. (DIDEROT, $1876: \mathrm{X}, 121)$. leur met dans les yeux de la vie, de la transparence, et l'humide, et le gras, et le nageant qui y est ; ils semblent vous regarder et vous sourire même de près ; seulement, à force de leur vouloir faire des chairs blanches et laiteuses, il les fait de craie.» (Ibidem, 206). Greuze cependant excelle dans sa peinture d'enfants : ce qu'il peint «c'est de la peau; c'est de la chair ; c'est du sang sous cette peau; ce sont les demi-teintes les plus fines, les transparents les plus vrais » (Diderot, 1876: XI, 444). Tout au long des Salons de 1761 à 1785, Greuze est le centre d'intérêt sans doute le plus grand. Dans le peintre, Diderot retrouve des thématiques et des valeurs importantes qu'il met en avant par un jugement esthétique au service du jugement moral.

volupté lorsqu'il est sensible aux corps. Et il s'étonne de l'indifférence des peintres: ceux-ci devraient être plus particulièrement attentifs aux corps des brunes:

les brunes piquantes comme nous en connaissons ont les chairs fermes et blanches, mais d'une blancheur sans transparence et sans éclat; c'est là ce qui les distingue des blondes, dont la peau fine, laissant quelquefois apercevoir les veines éparses en filets déliés et se teignant du fluide qui y circule, en reçoit en quelques endroits une nuance bleuâtre (Diderot, 1876 : X, 161).

Diderot fait également des remarques sur la sculpture, tout en faisant l'éloge de Falconet qui donne vie au marbre: "Quelle mollesse de chair! Non, ce n'est pas du marbre; appuyez-y votre doigt, et la matière qui a perdu sa dureté cèdera à votre impression. » ( Ibidem, 221).

L'art du portrait semble résider dans cette vraisemblance qui conjugue la perception du sang à circuler sous la peau et le caractère et la physionomie du personnage. Au contraire, le tableau qui semble invraisemblable est « un ouvrage vicié " (Diderot, 1876: XI, 12). C'est dans ce sens que Diderot attaque « le mélange d'êtres allégoriques et réels 》 (Ibidem, 310) qui « donne à l'histoire l'air d'un conte » : c'est ce défaut qui défigure la plupart des compositions de Rubens :

et voilà l'effet de tous ces sujets allégoriques empruntés de la mythologie païenne. Les peintres se jettent dans cette mythologie ; ils perdent le goût des événements naturels de la vie; et il ne sort plus de leurs pinceaux que des scènes indécentes, folles, extravagantes, idéales, ou tout au moins vides d'intérêt; car, que m'importe toutes les aventures malhonnêtes de Jupiter, de Vénus, d'Hercule, d'Hébé, de Ganymède, et des autres divinités de la fable ? (Ibidem, 57)

Cependant bien souvent, en voulant imiter, la composition des tableaux agit dans le sens inverse, c'est-à-dire que l'œuvre ne devient qu'une imitation grossière : les personnages sont maniérés, les vêtements s'affichent comme costumes, les personnages ne réalisent plus des actions mais jouent des rôles. Il n'y a plus de vie, de mouvement, mais une mise en scène théâtrale. C'est là l'une des grossières dérives de la théorie liant l'imitation au beau. L'excès de vraisemblable devient invraisemblance : "Tout ce qui est maniéré est faux ». Pour Jean Starobinski, la manière est «l'imagination affranchie de la subordination de la nature » (Starobinski, 1991: 36). Il est donc clair que, même si la 
critique peut se subordonner au peintre, le peintre, lui, ne peut pas se passer de la nature qu'il imite. En effet, il représente la nature en la peignant et ne fait pas l'inverse. Il ne peint pas la nature qu'il se représente.

Le bon peintre est justement celui qui connait si bien la nature qu'il peut en saisir les plus belles parties: "Mais pourquoi y a-t-il si peu d'hommes touchés des charmes de la nature? » (Diderot, 1876 : XI, 130), s'interroge Diderot. « C'est que la société leur a fait un goût et des beautés factices " (Ibidem, 130) : la mode dans les ateliers est le superflu, le déguisement, tout ce qui montre que les peintres du Salon de 1767 ont si peu de goût pour Diderot, qui parle dès l'adresse à Grimm de « la pauvreté de ce Salon-ci » (Ibidem, 4). Pour rester le plus proche possible de la nature, l'artiste prendra le modèle et lui ajoutera un trait de réel en l'enlaidissant, bien que la représentation reste sans personnalité.

C'est bien la présence d'un certain mauvais goût qui montre que le peintre n'est pas qu'imitateur. L'imitation de la nature ne suffit pas. Elle est, certes, la marque d'un certain «talent », d'une capacité à reproduire les beautés de la nature mais elles ne sont pas le signe de la capacité de discerner les belles parties de la nature. La recherche du beau idéal à travers un tableau implique donc un acte créateur. Si un tableau est beau, c'est d'abord parce que c'est une production de l'homme. Lors de la promenade Vernet, Diderot s'exclame: "sérieusement vous croyez que Vernet aurait mieux à faire que d'être le copiste rigoureux de cette scène» (Ibidem, 101). Contrairement à l'Abbé qui considérait que seul Dieu peut embellir la nature, Diderot s'affiche contre la position religieuse qui fait de la beauté des tableaux «l'ouvrage de Dieu» (Lojkine, 2007: 381). S'il est une méthode pour atteindre le beau idéal, ce n'est assurément pas en visant un idéal divin, bien au contraire l'esthétique en peinture repose sur les choix faits par l'artiste.

Dans le Salon de 1767, il constate que La Tour peint la nature comme elle est avec «le système de ses corrections telles qu'on les y voit tous les jours. »(Diderot, 1876 : XI, 151).

Diderot évolue dans sa vision sur l'art et la reproduction du réel. Diderot allait à l'encontre du public. À cette époque où la société prend de plus en plus de place, il faut des scènes tirées de la vie quotidienne, où elle retrouvera des personnages qui lui ressemblent de très près et qui emporteront d'emblée son adhésion.

4 Cependant, si dans ses premiers Salons, le philosophe exige que l'œuvre soit une copie fidèle et rigoureuse de la nature, il comprend petit à petit que toute reproduction intégrale est impossible. L'œil ne peut avoir qu'une vision partielle de la réalité. L'unité secrète de la nature échappe aux lois de l'observation. Il est impossible de cerner toute la diversité de cette nature que l'air et la lumière n'arrivent pas à réunir. Toute reproduction est pauvre et partielle. Le but de "copier» la nature sort déformée car l'artiste ne reproduit que des apparences du monde extérieur et une réalité illusoire.

Le travail du critique est également une production de l'homme et donc le texte des Salons semble être un traité critique, tentant de révéler le rôle de l'imitation et de la création au sein même du travail critique. L'imitation est avant tout une nécessité propre au travail du critique. Il se contraint au catalogue distribué à l'entrée du Salon qui fixe l'ordre des tableaux mais Diderot choisit librement la forme descriptive à chaque début de commentaire : de même les dimensions du tableau et les locutions " en haut ", " en bas ", " à gauche » visent à créer dans l'imaginaire du lecteur un tableau réel, ce qui correspond à l'imiter. 
26 Le recours à la première personne, toujours omniprésente, vise avant tout à faire percevoir au lecteur la même image que celle qui fut saisie par Diderot: l'auteur saisit dans l'instant de l'écriture un tableau dont il ne reste qu'une image personnelle.

Dans sa Lettre sur les sourds et muets, Diderot avait déjà pressenti que l'artiste peint d'après une autre dimension: "Le pinceau n'exécute qu'à la longue ce que l'œil du peintre embrasse tout d'un coup. » (Diderot, 1875 : I, 369). La conception de la beauté dépend d'un modèle intérieur patiemment élaboré où l'artiste réorganise et recrée les données de la réalité. Il traduit, transpose sa conception du Beau, devenu Beau artistique par un effort solitaire et patient. L'artiste projette sur l'œuvre son expression personnelle :

...manière de Poussin, de Le Sueur, du Guide, de Raphaël, des Carrache... manière de dessiner, d'éclairer, de draper, d'ordonner, d'exprimer ; toutes sont bonnes, toutes sont plus ou moins voisines du monde idéal (Diderot, 1876 : XI, 370) tout en se limitant au vraisemblable. À une époque où « le vrai dans la nature est la base du vraisemblable dans l'art » (Diderot, 1876 : XII, 107), l'artiste reste partiellement soumis à la nature, donnant à son génie le droit de s'exprimer et limitant son imagination.

L'espace littéraire donne lieu également à une véritable imagination expressive. Tout comme la littérature n'est pas un simple exercice de rhétorique, la peinture ne se réduit pas à un simple travail d'imitation.

30 Comme le peintre, le critique crée une nouvelle matière. C'est ici que Diderot opère une transfiguration littéraire de la peinture : les figures réelles sont transformées en figures abstraites, imaginaires, en fictions littéraires, en « tableaux absents qui se form[ent] dans sa pensée - c'est-à-dire sous sa plume » (Starobinski, 1991 : 61).

31 Le tableau, d'abord vu, ne se contemple plus de la même façon une fois le critique passé. Si l'on suit par exemple l'Accordée du village, de Greuze, Diderot après avoir explicité l'ordonnance, pur attrait visuel, explicite cette fois tout ce que semble traduire la peinture. Cette expression des passions se fait à des registres divers. Soit l'introduction d'un imaginaire littéraire justifie certains éléments du tableau (« Le moment qu'ils [les critiques] demandent est un moment commun, sans intérêt : celui que le peintre a choisi, est particulier » (Ibidem, 237), soit il révèle l'argumentation qui donne vie à la peinture : «Il faut avouer que tout cela est d'une convenance charmante avec la scène qui se passe, et avec le lieu et les personnages. Voilà un petit trait de poésie tout à fait ingénieux. » (Diderot, 1876 : X, 154). Par conséquent, le tableau devient sujet d'un espace romanesque car l'expression des passions mises en place, l'habilité du critique est de prouver sa théorie :

L'expression est en général l'image du sentiment. [...] L'homme entre en colère, il est attentif, il est curieux, il aime, il hait, il méprise, il dédaigne, il admire; et chacun des mouvements de son âme vient se peindre sur son visage en caractères clairs, évidents, auxquels nous ne nous méprenons jamais. (Ibidem, 484)

32 Le choix de ce tableau montre bien que le Salon présente tout un contexte social et historique. Le quotidien y est manifeste et soigneusement mis en scène. Dans ce tableau, les figures s'enchaînent, observait Diderot, et "vont en ondoyant et en pyramidant » ( Ibidem, 165) Mais cette construction rigoureuse donne pourtant une impression de naturel, et n'apparaît pas comme ayant été voulue ni même pensée par l'artiste. Les personnages figurés sur la toile sont crédibles.

L'inanimé devient animé, ou, pourrait-on dire, la peinture opère les différentes touches de pathétique, mêlé à la plume de l'auteur, créent une dimension nouvelle, une chaleur 
qui imprègne le spectateur, le caractère de la peinture l'emporte sur l'aspect purement esthétique, car la peinture s'identifie aux personnages:

L'expression exige une imagination forte, une verve brûlante, l'art de susciter des fantômes, de les animer [...] de les agrandir; l'ordonnance, en poésie ainsi qu'en peinture, suppose un certain tempérament de jugement et de verve, de chaleur et de sagesse, d'ivresse et de sens froid dont les exemples ne sont pas communs en nature. Sans cette balance rigoureuse, selon que l'enthousiasme ou la raison prédomine, l'artiste est extravagant ou froid. (Ibidem, 504).

Diderot appelle le portraitiste « l'historien » de la réalité ; il est donc le chroniqueur qui représente le réel à travers une vision subjective, distincte de l'imagination pusiqu'elle ne crée rien, « elle imite, elle compose, combine, exagère, agrandit, rapetisse. Elle s'occupe sans cesse de ressemblances» (Diderot, 1876: XI, 131). Il n'intéresse pas que le paysage soit vrai mais vraisemblable. Diderot admire Vernet précisément parce qu'il a su introduire la vérité dans le détail :

Que cela est beau, grand, varié, noble, sage, harmonieux, vigoureusement colorié ! Mille beautés éparses dans l'univers ont été rassemblées sur cette toile, sans confusion, sans effort, et liées par un goût exquis. C'est une vue romanesque dont on suppose la réalité quelque part. (Diderot, 1876 : XI, 106).

Toute œuvre d'art est un choix dont le tableau d'histoire ou les scènes de genre ne font pas exception. Parmi les figures à présenter, l'artiste doit se concentrer sur les éléments essentiels et éliminer les détails superflus malgré leur existence dans la réalité ou leur effet attendrissant. D'après Diderot,

quand on a le courage de faire le sacrifice de ces épisodes intéressants, on est vraiment un grand maître, un homme d'un jugement profond; on s'attache à la scène générale, qui en devient tout autrement énergique, naturelle, grande, imposante et forte. (Ibidem, 340).

En simplifiant sa toile, l'artiste fait le spectateur participer à son œuvre en la complétant par l'imagination. L'imitation de la nature débarrasse la représentation picturale qu'est la peinture de tout son superflu pour lui assurer un caractère de vraisemblance garant de la réussite de la représentation.

Moins l'œuvre sera achevée, plus elle suscitera l'intérêt du public. L'ébauche née d'un moment d'inspiration perd souvent son élan, sa vie à cause d'un long travail minutieux. Diderot affirme préférer une belle ébauche à un beau tableau, puisqu'étant « indéterminée, elle laisse plus de liberté à notre imagination, qui y voit tout ce qu'il lui plaît » (Diderot, 1876 : XI, 246).

L'artiste se sert de la ligne et de la couleur et c'est cette dernière qui touche le plus le philosophe, sensible à la sensualité chaude et vibrante des couleurs, à la richesse et diversité des tons d'un tableau: "C'est le dessin qui donne la forme aux êtres; c'est la couleur qui leur donne la vie. Voilà le souffle divin qui les anime» (Diderot, $1876: \mathrm{X}, 468$ ).

Voilà la raison pour laquelle le Salon de 1761 l'enchante :

Il me semble que nos peintres sont devenus coloristes. Les autres années le Salon avait, s'il m'en souvient, un air sombre, terne et grisâtre ; son coup d'œil cette foisci fait un autre effet. (Ibidem, 127)

Le coloriste prend le «soufflé divin » de la couleur pour recréer et modeler la réalité sur sa toile.

Chardin fascine Diderot par son travail de juxtaposition de couleurs et par des jeux de lumière : 
Ce sont des couches épaisses de couleur appliquées les unes sur les autres et dont l'effet transpire de dessous en dessus. (...) Approchez-vous, tout se brouille, s'aplatit et disparaît ; éloignez-vous, tout se recrée et se reproduit. (Ibidem, 195)

Seul le peintre talentueux, particulièrement doué, arrivera à harmoniser les tons les plus discordants parce qu'il maîtrise d'instinct le mélange des couleurs sur la palette.

Le sujet perd de l'importance face à l'audace et au charme de l'exécution qui produiront une œuvre où le Beau pictural s'imposera. Mais c'est le regard du (des) spectateur(s) qui recrée le tableau tout en réagissant à ce qu'il observe.

\section{Le spectateur dans les Salons}

Le spectateur dans le Salon de 1767 est d'abord une figure concrète, qui possède des référents définis, incarnés dans plusieurs catégories ou ensembles.

La situation énonciative de base du Salon de 1767 présente un locuteur Diderot qui s'adresse aux lecteurs de la Correspondance littéraire, destinataires finaux, à travers une adresse à Grimm.

Dans toutes les situations, le locuteur est Diderot lui-même, un spectateur connaisseur et écrivain qui fait le commentaire des centaines de toiles exposées au Salon. Il est toutefois un spectateur particulier, car sa mission n'est pas seulement de voir les tableaux mais d'en faire la critique. On note ainsi beaucoup d'occurrences du « je », car il remplit son rôle de critique en offrant son jugement à ses lecteurs. Il s'implique émotionnellement dans ces jugements: les verbes «aimer» et «préférer » expriment la sensibilité du critique de même que les exclamations.

de la Correspondance littéraire n'avaient pour la plupart pas l'occasion d'aller au Salon. Diderot tente de rendre par écrit ce qu'il voit et tente ainsi d'en faire des spectateurs, de dépasser leur statut de lecteurs, en relevant « le défi que le visible lance à la parole" (Berthet, 2006: 22). Le philosophe devait jouer avec deux publics antinomiques : d'une part, celui réel, connu, mais restreint des quelques accostumés de $L a$ Correspondance littéraire et, d'autre part, celui fantasmé, inconnu et ouvert de la postérité.

Diderot tour à tour s'exprime pour lui-même dans une opinion générale. Le locuteur reste Diderot mais il s'inclut dans l'ensemble des spectateurs. Le « je » devient un « nous ».

Loin de s'isoler de la masse des spectateurs, le jugement de Diderot épouse bien souvent un certain consensus. Dans l'introduction aux Euvres esthétiques, Paul Vernière écrit :

Information, intuition générale, souci d'être juste mais aussi d'être sincère,

d'exprimer le jugement du siècle, mais aussi de s'exprimer soi-même. Encore fallait-

il varier la mise en œuvre, utiliser la lettre, l'essai, le discours, le dialogue, et faire

de la critique d'art une œuvre d'art. (DIDEROT, $1988: 441$ )

Diderot ne se limite pas à présenter des spectateurs concrets dans un espace concret. Il crée des interlocuteurs non identifiés, des figures imaginaires de spectateur: «n'aimeriez-vous pas mieux cette tête coiffée d'humeur, sa draperie lâche et moins arrangée et son regard attaché sur le buste?» (Diderot, 1876: XI, 19-20). Les spectateurs imaginaires sont des constructions de Diderot pour servir son argumentation. Diderot crée des interlocuteurs fictifs, connaisseurs, capables de dialoguer avec lui. Le spectateur n'est que celui qui regarde, qui voit, il est également indissociable de la notion de jugement et d'expression d'un sentiment. 
51 La plupart du temps Diderot ne se contente pas de donner son avis mais il l'appuie de réactions d'autres spectateurs ou d'autres catégories de spectateurs dont il a pu observer ou entendre la réaction ou dont il imagine la réaction. L'introduction dans son texte du jugement d'autres spectateurs a deux justifications : d'une part, elle permet d'illustrer la thèse soutenue dans l'article "Beau» de L'Encyclopédie qui déclare qu'en donnant la parole à d'autres spectateurs, il montre qu'en effet le jugement du Beau est fonction de « rapports », que des spectateurs différents émettent des jugements différents; d'autre part, il semble vouloir faire de ces Salons des chroniques autant qu'une critique.

\section{Du langage pictural au langage littéraire}

Véritables comptes rendus des expositions de l'Académie de Peinture et de Sculpture, les Salons affrontent d'emblée la difficulté de transcrire les toiles par écrit. Pour Starobinski, cette difficulté est compensée par diverses stratégies discursives :

Pour qui sait goûter les stratégies du discours, les Salons sont d'une lecture passionnante : entre la soumission apparente au désir de l'ami éditeur, le francparler imprudent et vif, la visée implicite d'un public lointain, les conversations écoutées, rapportées, provoquées. Diderot exploite toutes les modalités de la communication verbale, comme pour compenser la difficulté fondamentale du passage de la peinture à la parole. (Starobinski, 1991 :14)

Starobinski évoque ainsi les enjeux fondamentaux de la Correspondance littéraire, chargée de tenir au courant les cours étrangères des événements artistiques français, en même temps que la principale difficulté qui se pose aux Salons: comment transcrire une exposition à laquelle les lecteurs n'ont pas participé ? Comment donner à voir des toiles par l'entremise du verbe?

Si à l'époque de Diderot, le monde de l'édition est déjà à même d'insérer vignettes et images aux textes, il n'en est pas encore question pour ce qui touche la Correspondance littéraire. Pour rendre compte des expositions du Salon carré du Louvre, Diderot est donc contraint de passer par la description des toiles qu'il a pu y contempler. En d'autres termes, l'écrivain n'a à sa disposition que le langage verbal pour donner à voir une multitude de tableaux. Au sein des Salons, apparaissent de nombreux parallèles entre ces deux arts que Diderot aime comparer : « La couleur est dans un tableau ce que le style est dans un morceau de littérature" (Diderot, 1876: 140). Cette phrase établit un parallélisme frappant entre deux arts fort différents a priori mais que Diderot, de par sa qualité de " littérateur ", " critique d'art ", cherche constamment à rapprocher. Pourtant ce rapprochement est difficile par l'inadéquation fondamentale des langages verbal et pictural. Les mots sont parfois insuffisants à rendre le réel car ils ne peuvent être les stricts équivalents des réalités qu'ils désignent. Le critique d'art se doit de dépasser, ou tout au moins de «compenser la difficulté fondamentale du passage de la peinture à la parole » (Starobinski, Ibidem). Diderot, dans son Essai sur la peinture, réaffirme la capacité de l'homme de lettres à se faire critique d'art : « Tout ouvrage de génie [...] est seul. On ne l'apprécie qu'en le rapportant immédiatement à la nature. Et qui est-ce qui sait remonter jusque-là ? Un autre homme de génie. » (Diderot, $1876:$ X, 520). Si peintre, sculpteur et homme de lettres ne pratiquent pas le même art, ils se retroyvent néanmoins dans leur " génie ", c'est-à-dire dans leur capacité à apprécier l'Art au sens global du terme. L'homme de lettres possède cette "facilité acquise par des expériences réitérées à saisir le vrai ou le bon, avec la circonstance qui le rend beau et d'en être promptement et 
vivement touché » (Diderot, $1876: \mathrm{X}, 519)$ et qui ne va cesser de s'accroître à mesure qu'il fréquente la peinture. Mais cette connaissance privilégiée ne suffit pas. Il lui faut encore pouvoir la partager de manière efficace avec son lecteur.

La difficulté fondamentale qui se pose pour Diderot est le public visé par la Correspondance littéraire : éditée en très peu d'exemplaires, elle se destine en effet aux cours étrangères qui apprécient particulièrement la culture française mais n'ont pas les moyens matériels de se rendre eux-mêmes à Paris pour voir les salons. L'objet des Salons est donc absent pour le lecteur mais aussi (ou du moins en partie) pour leur auteur. Ce dernier les rédige chez lui à partir du livret et des notes prises sur place. Connaissant clairement la difficulté de sa position de spectateur et de critique, Diderot se place dans la même position que ses futurs lecteurs et prend ainsi conscience de l'enjeu de ses descriptions : il doit faire vivre les salons et les toiles pou un public lointain et exigeant. Diderot met ainsi en branle la dialectique de la présence et de l'absence inhérente aux Salons, et que l'on retrouve dans sa tentative de ressusciter pour ses lointains lecteurs l'ambiance particulière du salon.

La description des toiles apparaît comme un préalable nécessaire à l'expression de la critique artistique. C'est pourquoi l'auteur apporte tout son soin à ce qu'Isabelle Vissière nomme le «texte-image " (Vissière, 1986: 111). Celle-ci relève différents procédés descriptifs et stylistiques que l'on retrouve dans les Salons et l'Essai sur la peinture. Diderot introduit fréquemment ces descriptions par le présentatif « Voici » présent dans le Salon de 1781. Avec lui, le présent de l'indicatif a pour effet d'actualiser les descriptions : instantanéité et simultanéité sont de mises dans les Salons car elles permettent de donner l'illusion de la présence de l'objet décrit: le tableau se donne ainsi à voir au lecteur comme au spectateur. Diderot fait ainsi littéralement vivre les tableaux qu'il décrit. L'hypotypose $^{1}$ des Marines de Vernet fait résonner la toile du bruit de la tempête : «on entend le bruit des flots, les cris de ceux qui périssent, on voit, on voit tout ce qu'il lui plaît » (Diderot, 1876: X, 99). Le pronom impersonnel permet d'englober le lecteur dans le spectacle total représenté sur la toile puisqu'il inclut le visuel et l'auditif. Dans d'autres toiles, le toucher et le goût sont également mis à contribution telles que les natures mortes de Chardin que Diderot admire en particulier. Touché par l'illusion du tableau, le spectateur est tenté de toucher les éléments qui y sont représentés, ce que cherche Diderot à rendre par l'écriture. L'auteur ne parvient pas à s'absenter de toute subjectivité. Diderot s'investit dans ses descriptions. Le portrait de Mme de Pompadour peint par Van Loo pour le Salon de 1759 fait l'objet d'une critique explicite qui se fond dans la courte description du tableau avant d'être explicitement formulée : les quelques détails donnés par Diderot sur la composition de ce portrait sont pour la plupart négatifs. Le vocabulaire péjoratif, sous la forme d'adjectifs, a une valeur dépréciative : c'est le cas du «visage » qualifié de "précieux», de la bouche de "pincée » et des mains qui sont celles «d'un enfant de treize ans» (Diderot, 1876: X, 92). Pourtant, objet de toutes les attentions au cœur de l'harmonie bleutée, la robe est une robe à la française, comble de l'élégance féminine au XVIII ${ }^{\mathrm{e}}$ siècle, dont la mode apparaît vers 1750.

57 À la description des toiles exposées, Diderot ajoute la critique à la fois positive et négative. La description n'est pas la fin première des Salons mais bien le moyen de l'expression d'un jugement esthétique.

58 Les réflexions de Diderot ne sont pas complètement coupées d'une certaine pratique de la peinture, propre au « littérateur ». Le motif de la réécriture des toiles occupe une grande partie des Salons qui ne se contentent pas de décrire les toiles exposées mais de les 
réinventer. En ce sens, les Salons ne sont pas de simples compte-rendus artistiques mais une véritable création.

L'art discursif de Diderot s'exprime très nettement dans les Salons et l'Essai sur la peinture où nous assistons à un véritable dialogue entre le critique d'art, ses différents lecteurs et son objet d'étude. L'écriture diderotienne réinvente l'art pictural par le biais du langage. Le passage du langage pictural au langage littéraire est un parcours difficile mais qui est ici surmontée de manière originale puisque Diderot ne cesse de faire le lien entre littérature et peinture.

C'est tout un travail sur l'art discursif qui fait des Salons, tout d'abord œuvre esthétique et jugement sur l'art, une chronique sur un événement qui n'était pas accessible à un public lointain qui avait un intérêt particulier pour la culture française. Les compte-rendus des Salons ressemblent à des histoires qu'on relit avec palisir ; des digressions pittoresques et vivantes réjouissent le lecteur et le tiennent sous le charme. Et parmi tout cela il y a des paysages devenus réels à ses yeux. Tantôt Diderot recompose la toile et dit ce qu'il aurait fait lui-même, tantôt le tableau devient prétexte à un récit ou à une évocation toute personnelle. Mais la toile de fond est souvent une actualité qui se montre par le biais de portraits, d'événements du quotidien et qui révèle des modes (et des modes de vie). Les Salons ne sont en fait qu'une pratique de la critique d'art et une chronique de l'actualité. Le spectateur est une composante indispensable de la réflexion de Diderot sur un dialogue avec l'œuvre d'art mais il se multiplie en différentes figures abstraites ou concrètes, de vrais interlocuteurs et instances regardantes des descriptions.

\section{BIBLIOGRAPHIE}

BERTHET, Dominique (2006). Les défis de la critique d'art. Paris : Kimé.

DIDEROT, Denis (1875). Cuuvres complètes (Vol. tome I). (J.Assézat, Ed.) Paris : Garnier Frères. DIDEROT, Denis (1876). CEuvres complètes (Vol. tome X). (J. Assézat, Ed.) Paris : Garnier Frères. DIDEROT, Denis (1876). CEuvres complètes (Vol. tome XI). (J. Assézat, Ed.) Paris : Garnier Frères. DIDEROT, Denis (1876). CEuvres complètes (Vol. tome XII). (J. Assézat, Ed.) Paris : Garnier Frères. DIDEROT, Denis (1876). Cuuvres complètes (Vol. tome XII). (J. Assézat, Ed.) Paris : Garnier Frères. DIDEROT, Denis (1988). CEuvres esthétiques. Paris : Garnier Frères.

FRIED, Michael (1990). La Place du spectateur. Esthétique et origines de la peinture moderne. Paris, Gallimard. «NRF Essais ».

LOJKINE, Stéphane (2007). L'Æil révolté: Les Salons de Diderot. Paris : Actes Sud. STAROBINSKI, Jean (1991). Diderot dans l'espace des peintres. Paris : Réunion des musées nationaux. VISSIÈRE, Isabelle (1988). « Une originalité de Diderot : le “ texte-image ” ». Recherches sur Diderot et l'Encyclopédie, no 4 . Aix-en-Provence. Université de Provence. 


\section{NOTES}

1. Une hypotypose est une figure qui regroupe l'ensemble des procédés permettant d'animer, de rendre vivante une description (http://www.etudes-litteraires.com/notions-stylistique.php\#3) au point que le lecteur « voit » le tableau se dessiner sous ses yeux. Il s'agit donc d'une figure de suggestion visuelle.

\section{RÉSUMÉS}

Les Salons de Diderot visent un public étranger qui ne pouvait pas fréquenter ces événements artistiques français. Ces spectateurs sont autant d'instances concrètes qu'abstraites, composante fondamentale de cette œuvre qui est en même temps un jugement esthétique et une chronique. Le critique d'art porte un regard aigu sur les toiles observées où le peintre «imite " la nature mais dans une prise de position tout à fait personnelle où cette nature est recréée d'après un modèle intérieur de l'artiste que le critique rapporte.

The Salons of Diderot are directed to a foreign audience who cannot visit these French artistic events. The audience is as much concrete as abstract instances, fundamental component of this work which is at the same time an aesthetic judgment and a chronicle. The art critic takes an acute look at the observed paintings where the painter «imitates " nature more in a personal position where this nature is recreated according to an internal model of the artist that the critic reports.

\section{INDEX}

Mots-clés : esthétique, imitation, réel, spectateur, art discursif

Keywords : aesthetics, imitation, reality, audience, discursive art

\section{AUTEUR}

\section{ANA FERNANDES}

Colaboradora do Centro de Línguas e Culturas / Universidade de Aveiro afpedroso[at]gmail.com 